\title{
Comunicación intercultural, contexto internacional y competencia traductora: una propuesta de mejora académica para los programas de traducción en Chile ${ }^{1}$ \\ Intercultural communication, international context, and translation competence: an academic improvement proposal for translation programs in Chile
}

Fecha de recepción: 6 de enero de 2020

Rosa ISABel BASAuRe CABero rosita.basaure@usach.cl Universidad de Santiago de Chile

Mónica del CARmen Ahumada Figueroa monica.ahumada@usach.cl Universidad de Santiago de Chile

Fecha de aceptación: 18 de enero de 2021

Resumen: La necesidad de comunicación no solo traspasa fronteras físicas, sino que también virtuales. En este sentido, la traducción ha sido siempre el medio para establecer dicho intercambio, y hoy más que nunca la realidad proyecta a los profesionales de la disciplina como agentes clave en la mediación intercultural entre realidades diferentes. Las instituciones académicas tienen conciencia del rol histórico de los traductores en el desarrollo de la comunidad internacional y de los requerimientos formativos que la realidad actual trae aparejados. No obstante, dentro de las líneas curriculares, en particular en el caso chileno, se evidencia que las áreas de competencia traductora relativas al conocimiento temático y el rol de la traducción en este contexto no es un aspecto prioritario, pese a que este tema se encuentra en la primera línea de la investigación disciplinar a nivel internacional. En este sentido, la investigación apunta a analizar esta problemática desde la perspectiva chilena, a fin de revisar los lineamientos curriculares desde lo académico-formativo, así como desde el desarrollo futuro de la investigación disciplinar. Metodológicamente, la investigación explora la relación entre la necesidad de comunicación intercultural de Chile y los planes de estudios de las instituciones, así como propone lineamientos

${ }^{1}$ Este artículo se enmarca en el proyecto de investigación DICYT N 031951BC, "El desafío de la comunicación intercultural en Chile: La mirada de las instituciones de Educación Superior que imparten la carrera de Traducción e Interpretación", de la Vicerrectoría de Investigación, Desarrollo e Innovación de la Universidad de Santiago de Chile.

Nuestros agradecimientos a la alumna asistente del proyecto, Lic.Romina Correa.

Hikma 20 (1) (2021), 25 - 44 
para la formación curricular y profesional de futuros traductores. Se presenta la reflexión respecto de la relación entre interculturalidad y competencia traductora para continuar con la revisión de los currículums chilenos y terminar con una propuesta que considera este aspecto como un eje formativo mediante el desarrollo de contenidos relativos al contexto de las relaciones internacionales dentro de la preparación profesional. El artículo concluye que un currículo flexible capaz de aportar conocimiento especializado permitirá a los futuros profesionales enfrentar los desafíos de un contexto sociocultural cambiante, propuesta que podría ser considerada en el resto de los programas académicos de la región.

Palabras clave: Formación de traductores, Competencia traductora, Relaciones internacionales, Chile.

Abstract: The need for communication not only crosses physical boundaries, but also virtual ones. In this sense, translation has always been the means to establish this interaction, and today more than ever reality projects the professionals of the field as key agents in the intercultural mediation among different realities. Academic institutions are aware of the translators' historical role in the development of the international community and the training requirements that current reality brings with it. However, within the curricular guidelines - particularly within Chilean case - it is evident that translation competence areas related to this context and the role of translation in this field is not a priority aspect, despite the fact that this topic is at the forefront of international disciplinary research. In this sense, this paper aims to analyze this issue from the Chilean perspective, in order to examine the curricular guidelines from the academic-training point of view as well as from the future development of disciplinary research. Methodologically, the research explores the relationship between Chile's need for intercultural communication and the educational curricula of the institutions and proposes guidelines for future translators in terms of curriculum and professional training. The relationship between interculturality and translation competence is presented to continue with the analysis of Chilean curricula and concluding with a proposal that considers this aspect as a formative axis by developing content related to the international relations context, as part of professional training. The article concludes that a flexible curriculum capable of providing specialized knowledge will allow future professionals to face the challenges of a changing socio-cultural context, proposal that could be considered by the rest of the academic programs in the region.

Keywords: Translators training, Translation competence, International relations, Chile. 


\section{INTRODUCCIÓN}

El lenguaje juega un papel fundamental en el modelamiento y desarrollo de cosmovisiones para un imaginario colectivo. Pero es mediante la traducción como eje articulador de la comunicación intercultural, que históricamente se han difundido las ideas fuerza de los movimientos sociales regionales e internacionales y vinculado sociedades por medio de un diálogo que puede alcanzar magnitud global.

Particularmente en las últimas décadas, debido a la masificación de las tecnologías de la información y la urgencia por transmitirla de manera rápida o inmediata, se ha reestructurado el rol de la traducción como mecanismo de interrelación entre los diversos actores de la Comunidad Internacional. Esto genera el desafío de revisar la importancia histórica del rol de la traducción como fenómeno articulador en las relaciones internacionales para establecer debates tales como el colonialismo, la migración, la protección global al medioambiente o la igualdad de género entre actores de la sociedad civil y los estados. Esta perspectiva implica además el posibilitar el desarrollo de capital humano avanzado especializado que comprenda y funcione bajo los mecanismos y las necesidades actuales de la comunicación intercultural (Diéguez, Lazo y Quezada, 2014).

A diferencia de otras regiones del mundo, en América Latina la discusión respecto del contexto cultural como un factor clave tanto en la práctica profesional evidencia carencias en cuanto a contenidos especializados que traten interculturalidad y relaciones internacionales de manera específica, lo que no deja de llamar la atención, pensando en una región en la cual las principales actividades productivas y áreas en donde la traducción se desarrolla como servicio tienen que ver con el intercambio de bienes y servicios, así como la llegada y salida de migrantes desde y hacia América Latina, aspectos donde la comunicación intercultural y la coyuntura política, cultural y económica son la base de la estabilidad o crisis de esta actividad laboral.

Este fenómeno ha resultado en que las instituciones latinoamericanas formadoras han comenzado a reevaluar su responsabilidad en el manejo de los aspectos sociales e interculturales de los profesionales de la disciplina, generando instancias de reflexión respecto al rol de la traducción en la comunidad internacional con el fin de abrir espacios de conocimiento en ámbitos concretos del currículo, a través de nuevas experiencias y prácticas pedagógicas en la materia.

Un ejemplo de este proceso de cambio corresponde al caso chileno, donde luego de los movimientos sociales por la equidad de género de 2018 , se desarrolló un renovado interés en incorporar alternativas curriculares y 
pedagógicas que fomentan el desarrollo de la competencia traductora mediante el aprendizaje de contenidos relativos al contexto internacional, específicamente al área de movimientos sociales y el impacto de aquello en la traducción (Basaure y Contreras 2019). Esto significó repensar nuevas instancias que permitan el comprender la historia de la traducción dentro de un contexto internacional donde permanentemente ocurren fenómenos sociales que confrontan los paradigmas culturales de las sociedades involucradas. No obstante, pese al avance, las instituciones de educación superior chilenas que preparan a los y las futuros profesionales evidencian una alineación parcial entre los contenidos de contexto internacional e interculturalidad y la formación actual respecto a comunicación intercultural desde esta perspectiva.

De esta manera, la investigación se plantea como un aporte a la ampliación de los enfoques fundamentalmente eurocéntricos que alimentan en la actualidad la disciplina, al considerar las particularidades de la actividad traductora en el contexto latinoamericano, por lo que como objetivo general se busca el determinar la relación entre los desafíos de comunicación intercultural y la formación de pregrado en Traducción e Interpretación para el caso chileno. Se plantea, asimismo, los siguientes objetivos específicos: 1) Explorar la relación entre la necesidad de comunicación intercultural de Chile y los currículums educativos de las instituciones; y 2) Proponer lineamientos para la formación curricular y profesional de futuros traductores de acuerdo a la situación chilena actual.

Respecto de la metodología, la primera parte del trabajo es de carácter exploratorio y considera las variables teóricas con el fin de articular el trabajo a partir de referencias bibliográficas y experiencia de expertos nacionales e internacionales respecto de la traducción y las relaciones internacionales para el caso chileno, lo que permite establecer una visión actualizada de la teoría disciplinar aplicada al rol articulador de los profesionales de la traducción y el medio tanto internacional como local.

La segunda parte es de carácter inductivo mediante el método de comparación constante, lo cual supone el análisis de una muestra representativa para el caso chileno de instituciones de educación superior que imparten programas de traducción e interpretación a partir de criterios objetivos y vigentes.

Además de la revisión de fuentes académicas oficiales (tales como las mallas curriculares que se encuentran disponibles en canales públicos y oficiales de difusión, perfiles de egreso, descriptores e informes de autoevaluación para acreditación nacional), esta sección consideró entrevistas de carácter semiestructurado con autoridades y académicos de 
las instituciones establecidas en la muestra, donde se abordó el factor de la multiculturalidad y la temática de las relaciones internacionales dentro del currículum.

En último término, la información levantada permite contar con un corpus de datos representativo desde una perspectiva traductológica que permite generar una propuesta curricular para la incorporación de estrategias de enseñanza para enfrentar temáticas relacionadas con el contexto internacional tanto en su formación como en la práctica disciplinar. Dichos lineamientos permiten que las propuestas curriculares respondan de manera más eficiente a los requerimientos de los profesionales de la traducción en su relación vincular con el exterior.

Como conclusión, se reflexiona respecto de la relevancia del contexto social de cara al rol de la traducción en la Comunidad Internacional para la formación profesional y se reafirma el factor de la interculturalidad y las relaciones internacionales como contenido relevante en el estudio, la práctica y la formación desde el punto de vista de la competencia traductora, lo que constituye una oportunidad para la actualización de líneas de formación, práctica e investigación de la traducción en América Latina a partir de la experiencia actual del caso chileno.

La relevancia de este estudio radica en la responsabilidad de generar procesos de actualización curricular permanente que permita a los y las estudiantes recibir una preparación adecuada al contexto socio cultural en el que desarrollarán su profesión, no solo pensando en un contexto local, sino que en uno global que constantemente enfrenta procesos sociales intercomunicados, y donde los diversos profesionales de la comunicación cumplen un papel preponderante.

1. EL ROL DEL CONTEXTO INTERCULTURAL EN LA FORMACIÓN PROFESIONAL TRADUCTORA

Analizar el concepto de interculturalidad requiere entenderse desde la diversidad de expresiones culturales, lingüísticas y espirituales que se entrecruzan al interior de un contexto internacional cambiante. Estas manifestaciones nos plantean la urgencia de enfrentar este tema desde la educación superior, donde los espacios para la reflexión no deben quedar como iniciativas individuales 0 institucionales, sino adquirir un espacio protagónico para ser parte del debate público respecto de la expresión de una realidad social donde se asume la interculturalidad presente. Es ahí donde consideramos que los profesionales de la traducción deben tener las herramientas e información necesarias que les permitan ser parte de dicha discusión. 
Catherine Walsh señala al respecto que cada vez es mayor la necesidad de promover las relaciones positivas entre distintos grupos culturales, de confrontar la discriminación, el racismo y la exclusión, de formar ciudadanos conscientes de las diferencias y capaces de trabajar por el desarrollo del país y en la construcción de una sociedad justa, equitativa, igualitaria y plural (Walsh, 2010). Desde esta perspectiva, todo esto se inscribe en el esfuerzo de generar iniciativas educativas al respecto, considerando que la visión del multiculturalismo sustentada en la idea de que cada cultura debe ser defensora de su propia identidad y que la educación debería responder a estos parámetros, hoy en día avanza hacia un concepto más amplio sobre interculturalidad que conduce a la idea de una interacción entre diferentes culturas, fortaleciendo la convivencia, y de alguna manera, esta nueva forma de entender los cruces culturales entre sociedades ayuda a configurar una nueva realidad global.

Por otra parte, Carlos Giménez Romero expresa que a los principios de igualdad y diversidad se debe agregar el principio de la interacción positiva, que permiten tanto a los niños como a los padres favorecer la cohesión social (Giménez Romero,2003). Esta también es la responsabilidad de las instituciones de educación superior para un mundo globalizado, en el sentido de que, dentro de la reflexión para la construcción y actualización curricular, es necesario considerar las herramientas y habilidades que necesitan los estudiantes durante su proceso formativo, para que luego, al desempeñarse como profesionales, enfrenten los desafíos que el mercado global de la traducción les impone, tales como la vinculación con profesionales de otras latitudes que poseen códigos culturales y sociales diversos; los desafíos que impone un contexto social y cultural interconectado, inmediato y global, y las particularidades que al mismo tiempo poseen los contextos locales tanto del mercado laboral como de la propia sociedad en la que se encuentran inmersos.

Bajo este escenario, se hace necesario el análisis de la experiencia pedagógica actual de los programas de traducción, considerando que a nivel mundial las instituciones educativas han integrado de diversa manera el factor del contexto internacional, así como las semejanzas y diferencias socioculturales al currículum a partir de incorporar la línea de actualidad internacional y comunicación intercultural desde diversas respuestas pedagógicas y didácticas a la formación de la competencia traductora de los futuros profesionales.

\subsection{Competencia traductora}

Para analizar la importancia de la formación en este aspecto, es necesario comprender que la competencia traductora -entendida como un 
subconjunto de competencias 0 habilidades que el estudiante debe desarrollar y fortalecer en el transcurso de su proceso de aprendizaje y durante su experiencia profesional (PACTE, 2018)- involucra competencias tales como la competencia comunicativa, referida a la comprensión en la lengua de partida y producción en la lengua de llegada; la competencia extralingüística, entendida como los conocimientos de la organización del mundo; la de transferencia, que tiene relación con el proceso de elaboración del texto final y el proceso de comprensión del contexto; la psicofisiológica, relativa a la activación de los recursos cognitivos que poseen los traductores; la competencia instrumental, que comprende las habilidades necesarias a nivel profesional; y, por último, la competencia estratégica como eje central del desarrollo profesional que permite resolver los problemas que conlleva la traducción (PACTE, 2018).

Por otra parte, Kelly (2002) precisa en su investigación el concepto de competencia traductora o macrocompetencia, y la define como «[...] el conjunto de capacidades, destrezas, conocimientos e incluso actitudes que reúnen los traductores profesionales y que intervienen en la traducción como actividad experta»(2002:14). Dicha definición incorpora así el espectro de habilidades necesarias para ejercer la traducción de manera eficiente y los elementos que la formación debe considerar a la hora de planificar el proceso de aprendizaje. Asimismo, se plantea la importancia de la formación destinada a desarrollar la subcompetencia cultural (que responde a los conocimientos que debe tener el profesional de la traducción respecto del contexto sociocultural tanto del texto de origen como en el texto meta), de la subcompetencia temática (que considera los conocimientos sobre temas específicos) y, por último, de la subcompetencia interpersonal ( la capacidad de relacionarse no solo con un equipo de trabajo, sino también con los clientes, sus requerimientos y características particulares) (Kelly, 2002).

Otros autores se refieren a la importancia de destacar el contexto y su relevancia para el desarrollo de capacidades verbales y no verbales (Canale, 1995) y, en este mismo nivel, incorporan el factor de la resolución de los conflictos comunicativos o subcompetencia estratégica, considerando tanto las competencias relacionadas con las funciones del lenguaje como con la adecuación de este lenguaje al contexto (Bachman, 1995).

En este mismo sentido, Márquez (2011) plantea la necesidad de flexibilizar el aprendizaje, no solo para fomentar la adaptación a nuevos equipos de trabajo, clientes o regulaciones, sino que también a la posibilidad de desempeñarse en las diversas temáticas, a partir de los requerimientos del mercado (Márquez, 2011), lo que también señala Kiraly en cuanto al refuerzo de la interculturalidad en la competencia traductora a partir de la incorporación tanto de la competencia interpersonal, relacionada con el 
trabajo en equipo, la tolerancia y empatía que se debe tener a la hora de relacionarse principalmente con el cliente y el usuario, como de la competencia cultural, que involucra conocer las tradiciones, los mitos, los valores, creencias y comportamientos de un país determinado (Kiraly, 2000).

Así, se plantea a nivel general la necesidad actual en la formación de traductores de alinear el currículum desde la perspectiva de los cambios que ocurren tanto en el mercado laboral como en el contexto en el que se desarrolla la sociedad en la que se encuentran inmersos dichos profesionales (Diéguez, Lazo y Quezada, 2014), por lo que es esencial considerar el desarrollo de competencias lingüísticas y de transferencia como ejes articulatorios de los programas de traducción, pero al mismo tiempo es vital el fortalecer las competencias asociadas a la resolución de problemas y comprensión del contexto temático y sociocultural, con el fin de preparar integralmente a los profesionales que deberán desarrollarse como mediadores en el diálogo intercultural.

\subsection{Interculturalidad en la formación traductora: El caso chileno}

Dado el análisis ya planteado, es necesario analizar el rol que les cabe a los profesionales de la traducción como actores relevantes en el proceso de transmisión de conocimientos y saberes a otra lengua. A esto debemos sumar el abordar los desafíos recientes frente a la comunicación intercultural.

El fenómeno migratorio, la globalización y la comunicación mediante plataformas virtuales nos exigen nuevas competencias que como veremos posteriormente, aún no han sido visibilizadas en los diferentes programas formativos chilenos. Dada esta realidad, esta investigación busca proponer la interculturalidad y el conocimiento respecto del contexto internacional como elementos articuladores en la formación académica.

Como hemos señalado, la formación de traductores profesionales en el caso particular de Chile se basa en la competencia traductora y las subcompetencias asociadas a la preparación práctica para el mercado laboral. Pero la traducción también se concibe como un medio para explicar y comprender las diferencias y similitudes entre individuos de diversas culturas, donde se considera no exclusivamente lo lingüístico, sino también lo social, cultural, político, filosófico y hermenéutico, pasando también por enfoques cognitivos, comunicativos, socioculturales e ideológicos (Hurtado Albir, 2001). Esta dualidad entre la práctica y la reflexión permite plantear que los traductores tengan un rol más activo en el espacio intercultural del discurso (Venuti, 2000), y donde es necesario tanto el desarrollo práctico de la disciplina como la reflexión teórica respecto de la importancia del rol social de la traducción, incluso como la única manera posible de transformar el mundo y provocar algo políticamente nuevo (Bhabha, 2002). 
En el caso de la situación académica de la traducción en Chile, ésta se sostiene a partir de un universo compuesto por un total de 3 programas conducentes al grado de técnico de nivel superior (Instituto Profesional Chileno Británico de Cultura, Universidad Arturo Prat e Instituto Profesional EATRI), con una duración promedio de 2 años; 1 programa en EATRI que confiere el título profesional de traductor, con una duración promedio de 4 años; y otros 12 programas que son licenciaturas (Pontificia Universidad Católica de Valparaíso, Universidad Católica de Temuco, Universidad de Atacama, Universidad de Concepción, Universidad de las Américas, Universidad Arturo Prat, Universidad de Artes, Ciencias y Comunicación UNIACC, Universidad de Playa Ancha, Universidad de la Serena, Universidad de Tarapacá, Universidad Tecnológica de Chile INACAP, Universidad de Santiago de Chile). Además, la oferta de programas de traducción en Chile incluye 4 programas de postítulo (Pontificia Universidad Católica de Valparaíso, Pontificia Universidad Católica de Chile, Instituto Profesional EATRI y Universidad Católica del Norte) y un único programa conducente al grado de Magíster en la especialidad (Pontificia Universidad Católica de Chile). Todos los programas mencionados se encuentran vigentes a noviembre 2019.

El análisis de la información se realizó a partir de la revisión de las mallas curriculares y los perfiles de egreso extraídos desde los sitios web públicos de todas las instituciones a partir de la 'búsqueda de las asignaturas y expresiones de interculturalidad presentes en los textos.

Asimismo, se priorizó la realización de entrevistas a académicos y autoridades a partir de los siguientes criterios de selección institucional: Instituto profesional EATRI, como la primera institución en establecer programas de traducción; Universidad de Tarapacá (por su situación geográfica que la hace una institución representante de las características de aquella zona productiva norte del país; Universidad de Santiago de Chile, por la diferenciación en la especialidad lingüística de su programa y como representante de la zona productiva centro del país, dada su situación geográfica; Universidad Católica de Temuco, por el desarrollo de vinculación con pueblos originarios, Universidad de Concepción, dada su ubicación y representante de la zona productiva sur del país; Universidad de Las Américas y Universidad Tecnológica de Chile INACAP, ambas por tener sedes en más de una ciudad Chile, y esta última por ser la institución con mayor cobertura geográfica con seis sedes a lo largo del territorio funcionando de manera simultánea y con programas diurnos y vespertinos. 


\subsection{Contexto sociocultural en las mallas curriculares}

En relación con los parámetros de este estudio, es posible establecer que pese a que en su mayoría, las instituciones chilenas declaran la relevancia de la relación traducción de cultura, éstas priorizan en el currículum la competencia lingüística de las lenguas de trabajo, la competencia instrumental en referencia a uso de tecnologías y la formación en competencias psicofisiológica y estratégica relativas a la autonomía y la gestión de proyectos por sobre los temas y contenidos relativos a interculturalidad y el rol de la traducción en las relaciones internacionales como actores sociales.

Al analizar los casos de la muestra, podemos establecer algunas características que resaltan. Por ejemplo, el Instituto Profesional EATRI pese a que impartió la asignatura de Actualidad mundial, ésta fue eliminada de la malla en la última revisión curricular (2015-2016) y actualmente no presenta en ninguno de sus programas referencia a contexto sociocultural en cuanto a actualidad nacional e internacional, relaciones internacionales, relaciones sociales interculturales o similares, ya sea en sus mallas, contenidos o en sus perfiles de egreso. En este caso, la alternativa pedagógica considerada por esta institución tras esa eliminación fue la incorporación de contenidos dentro de los textos de trabajo de las asignaturas del taller de traducción especializada enfocadas principalmente en la traducción económica y técnico-científica (Instituto Profesional EATRI, 2018).

Respecto la incorporación de contenidos interculturales dentro de cursos electivos, la Universidad de Santiago de Chile con el programa de Licenciatura en Lingüística aplicada a la Traducción ,(mención inglés y japonés e inglés y portugués), además de las asignaturas de Cultura en lengua inglesa, Cultura japonesa y Cultura portuguesa que se dictan para las respectivas menciones, ha realizado esfuerzos para incorporar cursos de contenido intercultural dentro de un sistema de electivos, donde destacan $E$ I rol del traductor en las relaciones internacionales y Traducción y estudios de género. Para contextualizar, se puede señalar que la primera se ha ofertado desde el año 2017 a la fecha y la última se ofertó en plan piloto durante 2018 , ambas para cuarto y quinto año de la actual malla curricular de la carrera. Cada asignatura declara noventa horas cronológicas en el semestre. (Universidad de Santiago de Chile, 2019)

En cuanto a la incorporación de interculturalidad interna, es decir, conocimiento respecto de los pueblos originarios, los casos que se destacan son la Universidad de Tarapacá y la Universidad Católica de Temuco.

Respecto del primero, la Universidad de Tarapacá hasta ahora integraba el factor cultural al currículum a partir de la asignatura «Literatura y

Hikma 20 (1) (2021), 25 - 44 
Cultura» en el sexto semestre. Sin embargo, el programa se encuentra en proceso de modificación curricular, en el que se está considerando incorporar diversos aspectos, incluido el fortalecimiento del factor de la enseñanza intercultural, no solo pensando en las relaciones internacionales y el fortalecimiento de la especialización técnica en el área productiva donde se encuentra la universidad (área norte-zona minera), sino también en relación al fortalecimiento de la vinculación con los pueblos originarios que habitan la zona (Universidad de Tarapacá, 2019).

En el caso de la Universidad Católica de Temuco, se evidencia el factor analizado en las asignaturas presentes en la malla curricular, tales como Historia socioeconómica nacional y regional y Lenguaje y construcción de mundo que se incluyen desde el primer semestre; Diversidad y globalización en el tercer semestre y los cursos llamados Electivos para la diversidad en el séptimo y octavo semestre (Universidad Católica de Temuco, 2019).

Cabe señalar que el desarrollo del programa de en la Universidad Católica de Temuco, en la región de la Araucanía, es diferente. Fundada en 1995, se renovó en 2009 manteniendo el Plan Vigente №2 con 10 semestres y trescientos créditos, encargándose de formar traductores del inglés hacia el español, en su calidad de especialistas. Al respecto, Ivette Carreño, traductora e investigadora de esta universidad señaló:

En el modelo educativo hay competencias genéricas propias de la Universidad y competencias específicas relativas a la carrera. En ellas se distinguen tres competencias, siendo la lingüísticacomunicativa, la sociocultural y la competencia extralingüística, entendida desde el traslado de la lengua de origen a la lengua meta. Todo esto desde la macro competencia de la traducción. Y la cuarta competencia es de la Facultad y nosotros estamos en el Departamento de Lenguas. En el Plan Vigente está "Pensamiento Crítico" y esta propende al espíritu creativo del profesional, del estudiante, y además tener una mirada sociocultural crítica a lo local. Saber cómo nuestro rol de traductor contribuye al ejercicio profesional. En la Malla de 2020, se plantea nuevamente el "Desarrollo del Pensamiento Crítico" y se disgregó desarrollándose en 3 niveles. En los niveles 1 y 2 se trabaja en 6 cursos que pertenecen al troncal anterior de la Facultad, abordando cinco áreas: teorías del desarrollo, lenguaje y construcción de mundo, diversidad, globalización, interculturalidad. Hoy la mirada no será desde diseño, sino hacer una revisión sociohistórica y adquirir nuevas miradas a aspectos relevantes de la profesión. El tercer nivel de la competencia está en el trabajo en clases en talleres de traducción y en la práctica profesional (I. Carreño, comunicación personal, 02 de agosto de 2019).

Hikma 20 (1) (2021), 25 - 44 
Al analizarse las diferentes realidades, se percibe que cada universidad cuenta con estrategias para abordar estas nuevas temáticas referidas a la interculturalidad, no obstante, pareciera ser la Universidad Católica de Temuco una de las más avanzadas al respecto. De hecho, en los nuevos programas para el 2020, se incorpora como un Proyecto Institucional, una impronta de carácter bicultural, desde donde todos los programas de estudio deberán sumarse a nuevas propuestas desde una mirada intercultural en particular hacia los pueblos originarios, dejando atrás enfoques que van contra una mirada multidimensional.

Por otra parte, otra alternativa para incorporar contenidos interculturales y actualidad internacional al currículum tiene relación con el integrar actividades extracurriculares tales como talleres o charlas de especialistas. Por ejemplo, el caso de la Universidad Tecnológica de Chile INACAP considera una malla curricular que incluye aspectos específicos del intercambio entre sociedades, particularmente en el contexto económico y de gestión, considerando la mención de negocios internacionales que declara.

Sin embargo, esta institución también se plantea un desafío de planificación curricular relevante en relación con que sus programas de estudio se desarrollan paralelamente en diversas sedes a lo largo del país, enfocados en la homogenización de evaluaciones y actividades pedagógicas. A este respecto, Loreto Serrano, especialista y asesora del Programa de Traducción entre los años 2012-2019 plantea que esto responde a «tratar de estandarizar lo más posible y que sea lo más homogéneo en el resultado del aprendizaje en todas las sedes» (Serrano, comunicación personal, 28 de noviembre de 2018), lo que deja poco margen a las modificaciones locales de las asignaturas que responden a distintas realidades contextuales geográficamente hablando y desde el punto de vista de las áreas productivas donde se desempeñarán los y las profesionales que egresan.

De todas maneras, se evidencia la intención de generar un currículum flexible o al menos modificable en el tiempo, considerando la presencia de aspectos del programa que tienen relación con los contenidos que se pueden incorporar o modificar ya sea por interés regional, consideraciones académicas o intereses de los alumnos. Es así como en la entrevista, la autoridad a cargo de los programas de traducción a nivel nacional señala que el contenido intercultural se incorpora mediante actividades extracurriculares tales como charlas o talleres que son coordinadas por sede, abiertas a toda la comunidad educativa «y es voluntario para cada alumno ir o no ir, pero sí hay una inquietud por incluir temas de actualidad» (Serrano, 2018). 


\subsection{El perfil de egreso como reflejo de la construcción curricular}

Las mallas curriculares actuales incorporan como parte fundamental del proceso enseñanza-aprendizaje, el tema de las prácticas profesionales y el perfil de egreso, siendo este último un factor clave al momento de determinarse la calidad del aprendizaje, las competencias adquiridas y las posibilidades de inserción laboral de los alumnos al momento de ingresar al mercado laboral. Sin embargo, es importante señalar la dificultad para sistematizar y comprobar dichos resultados a partir de la complejidad del seguimiento a los alumnos egresados, considerando además que en la realidad los alumnos van a desempeñarse en el marco las diversas posibilidades que les entrega la carrera de traducción.

Una visión interesante de la integración curricular del tema se evidencia a partir de la revisión de los perfiles de los programas chilenos, ya que al igual que las mallas, existen diversas estrategias de incorporación de temas de interculturalidad y/o multiculturalidad, a excepción del caso de EATRI, donde no se prioriza la interculturalidad, el contexto internacional o el estudios de fenómenos de impacto social tales como la equidad de género, mientras que se evidencia que la prioridad tiene relación con lograr la competencia traductora desde la perspectiva de la práctica y no de la reflexión traductora.

Asimismo, la Universidad de Tarapacá, con sede en Arica, considera el ingreso a un plan común de licenciatura con el programa académico de pedagogía en inglés, el cual potencia la competencia lingüística. El programa se divide al iniciar el 5to año entre Pedagogía y Traducción, para cubrir contenidos de cada disciplina específica, que para el caso de traducción se enfoca en potenciar la competencia tecnológica y orientarse hacia el desempeño profesional particularmente en agencias de traducción. Con respecto a la forma de abordar los temas socioculturales, Víctor Cámara, traductor y académico de la institución señaló:

No se consideran por la forma que se construyó la Malla Curricular ya que el enfoque está estrictamente relacionado con la traducción. Sin embargo, es la propia universidad en su Plan Estratégico quien obliga a trabajar con la cultura, con los pueblos aimaras, y nosotros esperamos con la nueva malla introducir cursos sobre interculturalidad y que no sean cursos exclusivos de inglés-español. La vinculación con la comunidad se ha pensado con charlas de historia, cultura anglosajona, cursos electivos [...] y en esto hay una profesora que da lengua, cultura y comunicación con una visión extranjera hacia lo local (V. Cámara, comunicación personal, 02 de agosto de 2019).

Con respecto a la Universidad Tecnológica de Chile-INACAP, el perfil de egreso se focaliza en la mención de Negocios Internacionales, pensando 
en las demandas del mercado laboral. Es así como debido a lo específico de esta mención, la malla consigna la asignatura Global Economics que entrega un contexto temático al futuro profesional, pero no se evidencia dicha especialización en el perfil de egreso al señalar que "el egresado estará en condiciones de "traducir textos del inglés al español que faciliten la comunicación entre culturas, conforme a estándares y normas de la profesión, mediante el uso integrado de herramientas tecnológicas» (Universidad Tecnológica de Chile INACAP, 2019)

Por su parte, la Universidad de Santiago de Chile a partir del año 1995, impartió la mención de inglés - japonés, dado el contexto internacional donde Japón se levantaba como segunda potencia mundial. A fin de que los alumnos pudieran responder a las necesidades laborales del momento e insertarse con su perfil de egreso en ese campo laboral específico, esto implicó interactuar con otros actores mediante la firma de acuerdos de cooperación con Japón. Años después, se incorporó la mención de traducción inglés-portugués en sintonía con las demandas lingüísticas de América del Sur y coincidiendo con la creación de MERCOSUR.

Siempre pensando en un contexto internacional cambiante, la enseñanza de la competencia traductora se fue ajustando a la contingencia nacional incorporando nuevas temáticas, lo que se alinea con el perfil declarado que establece: «El(la) traductor(a) profesional se caracteriza por la habilidad de transmitir mensajes escritos de una lengua a otra con fidelidad, manejando los léxicos y estilos requeridos. Además, demuestra un alto nivel de dominio de sus lenguas de trabajo y posee amplios conocimientos extralingüísticos y culturales, con una constante conciencia de la necesidad de actualización permanente» (USACH, 2020).

De acuerdo al análisis, es posible establecer entonces que, si bien los programas muestran preocupación por atender la competencia temática y extralingüística presentes en el enfoque intercultural de la disciplina y en la mayoría de ellos se observa el interés por la materia y la conciencia de que es necesario tratar de manera específica la comunicación intercultural en un contexto internacional desde se observa el desempeño laboral de la traducción, no se evidencia que estos contenidos se incorporen de manera constante, metódica y actualizada a las mallas curriculares y al perfil de egreso como un área prioritaria de la formación académica para hacer frente al interés de los estudiantes y a los cambios en el contexto disciplinar, social y laboral que deberán enfrentar en el futuro.

\section{UNA PROPUESTA CURRICULAR}

A partir del trabajo podemos establecer que los diversos programas académicos de traducción en Chile coinciden en lo plateado, es decir, aunque 
los contenidos especializados se incorporan al currículum a partir de consideraciones temáticas dentro de las asignaturas prácticas, mecanismos electivos o extracurriculares, no existe conciencia de la importancia que reviste la formación con hincapié en la sub competencia extralingüística relativa al contexto social internacional para entender las implicancias del escenario en donde los y las traductoras desarrollarán su labor profesional como entes activos en la comunicación intercultural, aportando en el desarrollo de la formación integral de los futuros profesionales.

Es así como se propone la flexibilización del currículum a partir de la implementación de un enfoque pedagógico que considere la interculturalidad como un eje formativo prioritario, específicamente en cuanto a considerar efectivamente la competencia traductora dentro de un contexto intercultural y como parte de la sociedad internacional, tanto en la reflexión como en la práctica traductológica.

Desde el punto de vista temático, la propuesta que cualquier modificación curricular debe promover la adaptabilidad de los programas no solo a las teorías y prácticas de la traducción, sino también al impartir conocimientos de la disciplina de las relaciones internacionales relacionadas a la interconexión global, siendo necesaria la formación en cuanto a conceptualización, contexto histórico y antecedentes relevantes, corrientes teóricas, conflictos y actores sociales estatales y no estatales, así como análisis de la contingencia y la vinculación del escenario global con las realidades locales.

Considerando la didáctica, se propone que los formadores sean facilitadores conscientes de un contexto internacional dinámico, propendiendo a la formación de las nuevas generaciones de profesionales a partir de un aprendizaje activo y autónomo de conocimientos y habilidades para el desempeño de su profesión, y que las instituciones formadoras consideren el facilitar el desarrollo de actividades pedagógicas, la promoción de líneas de investigación y la práctica de la traducción considerando aspectos interculturales y actualizando los programas a medida que el contexto internacional genera procesos de modificación social, ya sea que redunden en el plano internacional como en el local.

Basado en la investigación, se proponen tres alternativas de incorporación específicas, que pueden establecerse de manera conjunta o individual en el currículum, dependiendo de la flexibilidad de cada programa académico: 1) incluir contenidos a las asignaturas prácticas de traducción; 2) desarrollar actividades extracurriculares con profesionales especializados y 3) generar asignaturas relativas a la temática mencionada. 
En cuanto a la primera, es posible integrar el enfoque intercultural y las temáticas de relaciones internacionales a las unidades de estudio práctico o a las asignaturas de práctica traductológica, también conocidas como talleres. Dichas instancias plantean el desarrollo de talleres de traducción especializada donde se analicen textos de contenido internacional en relación con la preparación para el mundo laboral desde la perspectiva de lo técnico, al trabajar de manera individual y grupal en la producción de un texto final dentro de un ambiente controlado como es la sala de clases. Se espera que en las actividades los estudiantes también incorporen tecnología y nuevas plataformas en la aplicación práctica de recursos audiovisuales para la construcción de conocimiento, buscando formas de resolución de los problemas planteados mediante trabajo autónomo dentro y fuera del aula.

La segunda alternativa propone el desarrollo de actividades extracurriculares orientadas por un profesional de las relaciones internacionales que promueva que el estudiante interprete y modele los nuevos contenidos a partir de sesiones abiertas específicas que consideren contingencia internacional o la explicación de escenarios ya sea políticos, económicos o sociales. Dicha alternativa permite abrir los espacios académicos a la conversación con profesionales de esta área, lo que permitirá a los y las participantes comprender la coyuntura internacional y reflexionar respecto de sus propias creencias, las de sus pares y las de su entorno.

Por otra parte, y pese a que en la enseñanza superior existe el constante desafío de incorporar nuevos cursos debido al reducido tiempo que se cuenta para cada ciclo, los programas académicos establecen espacios de asignaturas electivas donde se abordan diversas áreas del conocimiento en estricta relación con las competencias, el perfil de egreso y la empleabilidad.

Es así como a partir de este trabajo, la tercera alternativa promueve la creación de asignaturas específicas que consideren esta realidad como prioritaria, desarrollando un diseño teórico-práctico basado en la generación de ciclos de aprendizaje activo y colaborativo (Sanmartí, 2002), así como de conocimiento reflexivo que dé cuenta de la relevancia de la relación entre contexto internacional y traducción, y donde la evaluación permita validar que los alumnos son agentes activos en un proceso de aprendizaje que les permita adquirir un conocimiento amplio y comprender los fenómenos sociales que ocurren en su entorno.

\section{CONCLUSIONES}

Hoy más que nunca se hace necesario profundizar en el rol de los traductores como mediadores interculturales en un mundo globalizado y en 
constante cambio. En este sentido, como parte de la formación de estos profesionales es necesario establecer lineamientos curriculares que deben estar explícitos en mallas y perfiles de egreso, con el fin de establecer las dinámicas de aprendizaje que luego en la práctica no solo generen un traspaso mecánico de información con una mirada superficial de su contexto social, sino que posibiliten que los y las estudiantes y futuros profesionales comprendan que el discurso traducido es parte del contexto histórico, social, económico y político de los pueblos.

En este sentido, pese a la importancia de la vinculación de la traducción desde la interculturalidad, así como el impacto de su labor en la realidad de las relaciones entre actores internacionales y locales, al menos para el caso chileno no se han incorporado a los programas de estudio ni estos contenidos ni la reflexión al respecto de una forma sistemática y prioritaria, quedando en desmedro con respecto a la discusión disciplinar global.

Resulta pues, que los resultados de esta investigación todos los programas chilenos consideran las dimensiones de la competencia traductora mencionados en este estudio, priorizando la competencia lingüística de las lenguas de trabajo, la competencia instrumental en referencia a uso de tecnologías y la formación en competencias psicofisiológica y estratégica relativas a la autonomía y la gestión de proyectos, aunque el establecer la relevancia del aspecto internacional de la formación dentro de la competencia extralingüística queda supeditado a un segundo plano.

Esta evidencia nos plantea el generar modificaciones curriculares que permitan a los futuros profesionales enfrentar los desafíos de un contexto sociocultural cambiante, mediante mecanismos que permitan actualizar contenidos, estrategias pedagógicas y programas de las asignaturas para desarrollar un currículo flexible capaz de aportar conocimiento especializado y que considere los cambios constantes de un mundo global.

Dada la relevancia del contexto sociocultural en el desarrollo de la competencia traductora, es necesario concientizar a las instituciones educativas, a estudiantes y a profesionales dedicados a la traducción que constituyen la base de generación del capital humano avanzado mediador que será agente clave en el desarrollo integral de las relaciones internacionales de los próximos años y de las mejoras de las realidades locales gracias a la cooperación internacional.

Finalmente, podemos decir que en una época de cambios y donde se levantan movimientos sociales locales e internacionales que afectan el desarrollo humano en diversos ámbitos, es necesario que los traductores asumamos nuestra responsabilidad en la sociedad global y podamos 
construir los lazos interculturales que sustenten un mundo que funciona en un contexto de cambio y bajo diversas perspectivas culturales.

\section{REFERENCIAS BIBLIOGRÁFICAS}

Bachman, L. (1995). Habilidad lingüística comunicativa. En Llobera et al. (Coord.), Competencia comunicativa. Documentos básicos en la enseñanza de lenguas extranjeras. Madrid: Edelsa.

Basaure-Cabero, R. I. y Contreras-Torregrosa, M. (2019). Movimientos feministas y enfoques de género: integración del debate en las líneas curriculares para la formación de traductores en Chile. Mutatis Mutandis, 12(1), 156-181. Doi: 10.27533/udea.mut.v12n1a06

Bhabha, H. (2002). El lugar de la cultura. Buenos Aires: Manantial.

Cámara, V. Académico Universidad de Tarapacá. Entrevista realizada para esta investigación el 02 de agosto 2019.

Canale, M. (1995). De la competencia comunicativa a la pedagogía comunicativa del lenguaje. En Llobera et al. (Coord.). Competencia comunicativa. Documentos básicos en la enseñanza de lenguas extranjeras. Madrid: Edelsa.

Cámara, V. Académico Universidad de Tarapacá. Entrevista realizada para esta investigación el 032 de agosto 2019

Carreño, I. Académico Universidad Católica de Temuco. Entrevista realizada para esta investigación el 02 de agosto 2019.

Diéguez, M.I, Lazo, R. M. y Quezada, C (2014). Estudio de mercado de la traducción en Argentina, Chile y España: perfil académico y profesional de los traductores. Onomázein, Revista semestral de lingüística, filología y traducción 30, 70-89. Recuperado de http://onomazein.letras.uc.cl/Articulos/N30/30_6_Dieguez_FINAL.pdf

Giménez Romero, C. (2003). Pluralismo, multiculturalismo e interculturalidad. Rev. Educación y Futuro №8. p11-20. Recuperado de https://dialnet.unirioja.es/servlet/articulo?codigo=2044239

Hurtado, A. (2001). Traducción y Traductología: Introducción a la Traductología. Madrid: Cátedra.

Instituto Profesional EATRI (2018). Quiénes somos. Instituto Profesional EATRI. Recuperado de http://www.eatri.cl/quienes-somos/

Kelly, D. (2002). Un modelo de competencia traductora: bases para el diseño curricular. Puentes. Hacia nuevas investigaciones en la mediación 
intercultural, 1, 9-20. Recuperado de http://wpd.ugr.es/ greti/revistapuentes/pub1/02-Kelly.pdf

Kiraly, D. (2000). A Social Constructivist Approach to Translator Education. Empowerment from Theory to Practice. Manchester: St. Jerome Publishing.

Márquez, D. (2011). Las once competencias del traductor: el perfil ideal en servicios de traducción. Comunicación, cultura y política. Revista de Ciencias Sociales, 4, julio-diciembre 2011, 53-68. Recuperado de https://journal.universidadean.edu.co/index.php/revistai/article/view/65 9

PACTE (2018). Competence levels in translation: working towards a European framework. The Interpreter and Translator Trainer, 12(2), 111-131. Recuperado de https:// ddd.uab.cat/record/194868

Pym, A. (1998). Method in Translation History. Manchester: Saint Jerome Publishing.

Sanmartí, N. (2002). Organización y secuenciación de las actividades de enseñanza/ Didáctica de las ciencias en la educación secundaria obligatoria. Madrid: Síntesis Educación.

Sepúlveda, P. (2018, 20 de mayo). Feminismo chileno vive su revolución más importante en 40 años. La Tercera. Recuperado de http://www.latercera.com/tendencias/noticia/feminismo-chileno-viverevolucion-mas-importante-40-anos/172111/

Serrano, L. Coordinadora nacional programas de traducción Universidad Tecnológica INACAP. Entrevista realizada para esta investigación en enero 2019.

Stufflebeam, D.L. y Shinkfield, A.J., (1987). Evaluación sistemática. Guía teórica y práctica. Barcelona: Paidós-MEC.

Universidad Católica de Temuco (2018). Admisión. Traducción. Recuperado de https://admision.uct.cl/traduccion/

Universidad de Tarapacá (2018). Admisión. Licenciatura en Inglés. Recuperado de https://admision.uta.cl/index.php/licenciatura-eningles/

Universidad de Santiago de Chile (2019). Licenciado en Lingüística aplicada a la traducción mención en inglés-japonés 0 inglés-portugués. Recuperado de http://www.fahu.usach.cl/licenciatura-en-linguisticaaplicada-la-traduccion-en-ingles-japones-e-ingles-portugues 
Universidad Tecnológica de Chile INACAP (2019). Traductor Inglés-Español, Mención Negocios Internacionales Recuperado desde http://www.inacap.cl/tportalvp/humanidades-yeducacion/contenido/carreras/traductor-ingles-espanol-mencionnegocios-internacionales

Venuti, L. (2000). The Translator's Invisibility: A History of Translation. Londres. Routledge.

von Flotow, L. (Ed.) (2011). Translating Women. Ottawa: University of Ottawa Press

Walsh,C. (2010) Interculturalidad crítica y educación intercultural en Viaña, J, Tapia, L. y Walsh, C. Construyendo Interculturalidad Crítica. Instituto Internacional de Integración, Convenio Andrés Bello. Recuperado de https://aulaintercultural.org/2010/12/14/interculturalidad-critica-yeducacion-intercultural/ 